\title{
Outcome after Simultaneous Resection of Gastric Primary Tumour and Synchronous Liver Metastases: Survival Analysis of a Single-center Experience in China
}

\author{
Qian Liu, Jian-Jun Bi, Yan-Tao Tian, Qiang Feng, Zhao-Xu Zheng, Zheng Wang*
}

\begin{abstract}
Background: The optimal surgical strategy for the treatment of synchronous resectable gastric cancer liver metastases remains controversial. The aims of this study were to analyze the outcome and overall survival of patients presenting with gastric cancer and liver metastases treated by simultaneous resection. Materials and Methods: Between January 1990 and June 2009, 35 patients diagnosed with synchronous hepatic metastases from gastric carcinoma received simultaneous resection of both primary gastric cancer and synchronous hepatic metastases. The clinicopathologic features and the surgical results of the 35 patients were retrospectively analyzed. Results: The 5-year overall survival rate after surgery was $14.3 \%$. Five patients survived for more than 5 years after surgery. No mortality has occurred within 30 days after resection, although two patients (5.7\%) developed complications during the peri-operative course. Univariate analysis revealed that patients with the presence of lymphovascular invasion of the primary tumor, bilateral liver metastasis and multiple liver metastases suffered poor survival. Lymphovascular invasion by the primary lesion and multiple liver metastases were significant prognostic factors that influenced survival in the multivariate analysis $(p=0.02, p=0.001$, respectively $)$. Conclusions: The presence of lymphovascular invasion of the primary tumor and multiple liver metastases are significant prognostic determinants of survival. Gastric cancer patients without lymphovascular invasion and with a solitary synchronous liver metastasis may be good candidates for hepatic resection. Simultaneous resection of both primary gastric cancer and synchronous hepatic metastases may effectively prolong survival in strictly selected patients.
\end{abstract}

Keywords: Gastric cancer - liver metastases - hepatectomy - prognosis

Asian Pac J Cancer Prev, 16 (4), 1665-1669

\section{Introduction}

Gastric carcinoma is the major cause of cancer related deaths in China (Zhang et al., 2014). Due to the unspecific symptoms at an early stage and lack of a screening schedule, the majority of patients with gastric cancer are diagnosed at an advanced stage. Liver is a frequent organ of distant metastases from gastric cancer, and the incidence of liver metastases from primary gastric cancer ranges from 5 to $9 \%$ (Thelen et al., 2008).

The effect of surgical resection of liver metastasis from gastric cancer remains unclear. A key question was whether simultaneous resection of liver metastases provided any long-term survival benefit. Because patients with liver metastases from gastric carcinoma usually associated with extrahepatic disease, such as peritoneal dissemination, lymph node metastasis, and direct cancer invasion of other organs (Ambiru et al., 2001; Morise et al., 2008; Tiberio et al., 2009), only a few of them are candidates for hepatic resection. There is increasing evidence in the literature that hepatectomy for metastases from gastric cancer can improve survival with a 5-year survival rate of 10-38\% (Okano et al., 2002; Sakamoto et al., 2003; Shirabe et al., 2003; Thelen et al., 2008; Tsujimoto et al., 2010), but few studies have addressed the value of surgical treatment in patients with gastric primary tumour and synchronous liver metastases.

Accordingly, we performed the present retrospective study to evaluate the outcomes of patients undergone simultaneous resection of gastric primary tumour and synchronous liver metastases and to identify the prognostic factors and the actual benefits of surgical therapy.

\section{Materials and Methods}

Institutional review board approval was obtained before conducting this study. From January 1990 to June 2009, 35 consecutive patients underwent simultaneous gastrectomy and hepatectomy for synchronous hepatic metastases from gastric cancer at the Department of 
Abdominal Surgical Oncology, Cancer Hospital of the Chinese Academy of Medical Sciences, Peking Union Medical College. Our indications for resection of synchronous hepatic metastases from gastric cancer were basically as follows: $(i)$ curative resection of the primary gastric lesion; (ii) acceptable hepatic functional reserve and postoperative hepatic function; (iii) macroscopically complete resection of primary tumor and hepatic metastases; ( $i v)$ no signs of peritoneal dissemination or any other distant metastases; $(v)$ patients with complete follow-up inforemation. Cases with synchronous en bloc resections of gastric cancer directly invading the liver were excluded. No patient has received neoadjuvant therapy before surgery. All patients gave written informed consent before hepatectomy.

All the patients routinely underwent a upper gastrointestinal radiography, chest X-ray, abdominal ultrasonography, computed tomography of the abdomen and pelvis, or positron emission tomography scanning and electronic gastroscope examination before surgery to identify the disease region and the pathologic type. All patients diagnosed with gastric adenocarcinomas after pathologic examination.

All patients underwent radical subtotal or total gastrectomies, depending on the tumor location and intraoperative verification of tumor-free resection margins, as well as D2 lymphadenectomies. The methods of reconstruction after distal gastrectomy include Billroth I, II or Roux-en-Y. Roux-en-Y reconstruction and jejunal interposition are the most common methods used after a total gastrectomy. The surgical procedure of hepatectomy was classified as anatomic resection (segmentectomy and lobectomy) or limited resection (all resections less extensive than segmentectomy).

The patients' clinical characteristics, histopathological parameters and follow-up data were recorded and investigated. All resected specimens were histopathologically confirmed and staged according to the seventh edition of the American Joint Committee on Cancer (AJCC) mamual. In patients with multiple hepatic metastases, the pathologic findings of the largest tumor were taken as representative of those of the other tumors, because all the tumors showed similar pathologic features. All patients received macroscopically complete (R0 or R1) hepatic resection. $\mathrm{R} 0$ resection was defined by no residual tumor and R1 was defined by microscopically positive surgical margins. Postoperative adjuvant chemotherapy included a docetaxel/cisplatin/5- fluorouracil (DCF) regimen, an oxaliplatin or cisplatin/5-fluorouracil regimen, and an irinotecan/cisplatin regimen.

Patients were examined every three months for the first two years and every six months thereafter. The followup protocol involved standardized clinical examinations, including gastroscopy, chest X-ray, abdominal and pelvic ultrasound or CT or magnetic resonance imaging or positron emission tomography and tumor markers. All patients were followed-up until death or the cutoff date (May 31, 2014). The median follow-up time was 41 months (range, 12-85). Only deaths due to gastric cancer were considered. Deaths due to other causes were censored at the date of death. Follow-up evaluation was implemented by means of outpatient service, telephone calls, and letters.

\section{Statistical analysis}

Survival data for the 35 patients were analyzed using the Kaplan-Meier method. The log rank test was used to assess the differences between prognostic factors. A multivariate analysis of the factors influencing the 5-year survival rate was performed using the Cox proportional hazards model. $p<0.05$ was considered statistically significant. Statistical analyses were performed with SPSS software, version 15.0 for Windows (SPSS Inc., Chicago, IL, USA).

\section{Results}

The study involved 22 men and 13 women, with a male:female ratio of 1.69:1. The median age of 35 patients was 56 years (34-77 years). Fifteen patients had a tumor diameter less than $5.0 \mathrm{~cm}$, and other twenty patients had a tumor diameter greater than or equal to $5.0 \mathrm{~cm}$. The location of the primary gastric cancer was the upper and middle parts in 10 patients, lower part in 25 patients. The depth of the tumor was T1-2 in 6 patients, and T3-4 in 29 patients. Of the 35 patients, metastatic lymph node involvement was present in $31(88.6 \%)$. The identified tumors of fifteen patients were well and moderate differentiated, and other twenty patients had poorly differentiated adenocarcinoma of the stomach. Twenty patients had lymphovascular invasion. The surgical procedures were simultaneous resections of the primary tumor and synchronous hepatic metastases. Hepatic resection was indicated for patients who did not have peritoneal dissemination or any other distant metastases (restricted to the liver). The surgical procedures used for the primary tumors included distal gastrectomy in 27 patients, total gastrectomy in 6 patients, and proximal gastrectomy in 2 patients. 24 patients had the maximum tumor diameter of liver metastases less than or equal to $2 \mathrm{~cm}$, and other 11 patients had the maximum tumor diameter greater than $2 \mathrm{~cm}$. The distribution of the tumors was unilobar in 30 patients and bilobar in 5 patients. 27 patients had a solitary tumor and 8 patients had multiple (2-6) tumors. The hepatectomy procedure consisted of non-anatomic limited resections in 25 patients, segmentectomy in 4 patients, left hemihepatectomy in 3 patients, and right hemihepatectomy in 3 patients. In thirty patients the resection was classified as curative, whereas five patients had palliative resection with microscopical hepatic resection margin positive. Postoperative chemotherapy to all patients, and postoperative radio-frequency ablation to five patients.

In-hospital and 30 day postoperative mortality was zero. Two patients developed complications, which comprised intestinal leakage in one and bile leakage in the other. Postoperative complications were treated conservatively. The overall morbidity rate was $5.7 \%$. Microscopical curative resection was accomplished in 30 patients $(85.7 \%)$. The median overall survival time of the patients was 33 months and the overall 5-year survival rate was $14.3 \%$. Five patients are tumor-free and survived 
for more than 5 years after the hepatectomy. Twentyfour patients out of the thirty-five $(68.6 \%)$ developed recurrence in the remaining liver, four $(11.4 \%)$ in the lung, and two (5.7\%) in the lymph nodes, with an overall recurrence rate of $85.7 \%(n=30)$ during the follow-up period. None of the patients underwent a second hepatic resection for recurrent intrahepatic metastases, and systemic chemotherapy was administered in 24 out of the 30 patients with recurrent tumors. Factors associated with survival, as determined by Kaplan-Meier and log rank analysis, are presented in Table 1. Univariate analysis revealed the following factors to be associated with poor survival: presence of lymphovascular invasion of the primary tumor $(p=0.01)$; bilateral metastasis $(p=0.03)$; multiple metastasis $(p=0.003)$ (Figure1). No survival difference was found between patients underwent R0 and R1 hepatectomy. The multivariate Cox proportional regression analysis showed that lymphovascular invasion of the primary gastric tumor and multiple metastasis were

Table 1. Clinicopathological Factor Associated with the Overall Survival of Patientswith Liver Metastases from Gastric Cancer after Hepatectomy

\begin{tabular}{|c|c|c|c|c|}
\hline Factor & $\mathrm{n}$ & $\begin{array}{l}\text { Median } \\
\text { survival time } \\
\text { (months) }\end{array}$ & $\begin{array}{l}\text { 5-year } \\
\text { survival } \\
\text { rate }(\%)\end{array}$ & p-value \\
\hline \multicolumn{5}{|l|}{ Gender } \\
\hline Male & 22 & 35.5 & 13.6 & 0.86 \\
\hline Female & 13 & 30 & 15.4 & \\
\hline \multicolumn{5}{|l|}{ Age(years) } \\
\hline$\leq 60$ & 18 & 28 & 16.6 & 0.98 \\
\hline$>60$ & 17 & 40 & 11.7 & \\
\hline \multicolumn{5}{|l|}{ Primary gastric tumors } \\
\hline \multicolumn{5}{|l|}{ Tumor size $(\mathrm{cm})$} \\
\hline$<5 \mathrm{~cm}$ & 20 & 43 & 20 & 0.14 \\
\hline$\geq 5 \mathrm{~cm}$ & 15 & 20 & 6.7 & \\
\hline \multicolumn{5}{|l|}{ Location } \\
\hline Upper/Middle & 12 & 20 & 16.7 & 0.85 \\
\hline Lower & 23 & 35.5 & 13 & \\
\hline \multicolumn{5}{|l|}{ Depth of invasion } \\
\hline $\mathrm{T} 1-2$ & 6 & 44.5 & 16.7 & 0.3 \\
\hline T3-4 & 29 & 30 & 13.8 & \\
\hline Lymph node metastasis & & & & 0.66 \\
\hline Absent & 4 & 45 & 25 & \\
\hline Present & 31 & 30 & 16.1 & \\
\hline Histologic differentiation & & & & 0.32 \\
\hline Well/ Moderate & 14 & 40.5 & 21.4 & \\
\hline Poor & 21 & 26 & 9.5 & \\
\hline lymphovascular invasion & & & & 0.01 \\
\hline Absent & 16 & 44.5 & 25 & \\
\hline Present & 19 & 22 & 5.3 & \\
\hline \multicolumn{5}{|l|}{ Liver metastases } \\
\hline \multicolumn{5}{|l|}{ Tumour size $(\mathrm{cm})$} \\
\hline$\leq 2 \mathrm{~cm}$ & 24 & 34 & 8.3 & 0.37 \\
\hline$>2 \mathrm{~cm}$ & 11 & 33 & 27.3 & \\
\hline \multicolumn{5}{|c|}{ Hepatic tumor distribution } \\
\hline Unilateral & 30 & 39 & 16.7 & 0.03 \\
\hline Bilateral & 5 & 17 & 0 & \\
\hline \multicolumn{5}{|l|}{ Tumor number } \\
\hline Solitary & 27 & 40 & 18.5 & 0.003 \\
\hline Multiple & 8 & 18.5 & 0 & \\
\hline \multicolumn{5}{|l|}{ Hepatic resection margin } \\
\hline R0 & 32 & 30 & 12.5 & 0.47 \\
\hline $\mathrm{R} 1$ & 3 & 48 & 33.3 & \\
\hline
\end{tabular}

Table 2. Multivariate Analysis of Predictive Factors Contributing to Survival in Gastric Cancer Liver Metastases Patients

\begin{tabular}{lcll}
\hline Factor & Odds ratio & 95\% C.I. & p-value \\
\hline Lymphovascular invasion & 1.749 & $1.432-8.167$ & 0.02 \\
Multiple metastasis & 2.528 & $1.061-10.371$ & 0.001 \\
\hline
\end{tabular}

A

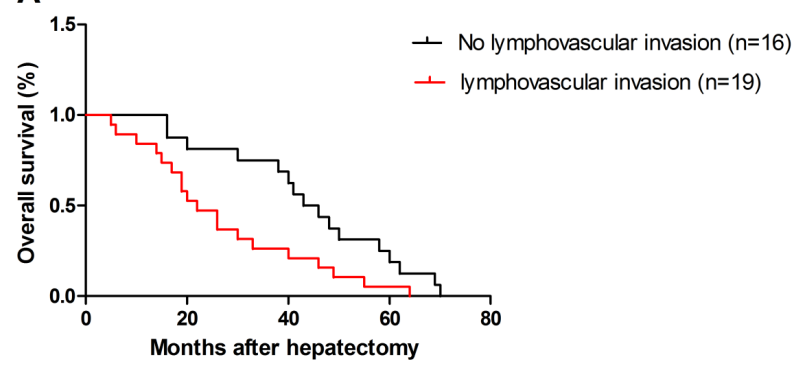

B

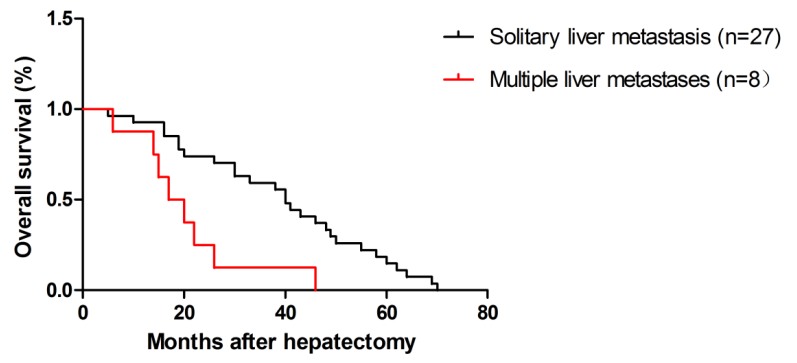

Figure 1. A: Overall Survival as Assessed by Kaplan-Meier Analysis According to the Presence of Lymphovascular Invasion of the Primary Lesion. B: Kaplan-Meier Survival Analysis of Overall Survival According to the Number of Hepatic Metastases

independent risk factors with poor survival. ( $p=0.02$ and $p=0.001$, Table 2).

\section{Discussion}

Whereas liver metastases develop in approximately $50 \%$ of all patients with colorectal carcinoma, the incidence of liver metastases from primary gastric cancer ranges from 5 to $9 \%$. Although hepatic resection has been widely accepted as a potentially curative approach for patients with liver metastases from colorectal carcinoma, with a 5-year survival rate of 30\%-50\% (Yasui et al., 1997; Okano et al., 1999; Mann et al., 2008), the significance of hepatic resection for metastases from gastric carcinoma is still controversial. Because of multiple bilateral metastases, extrahepatic diseases, or advanced cancer progressions such as peritoneal dissemination and extensive lymph node metastases, only $10 \%-20 \%$ of patients with hepatic metastases from gastric carcinoma are suitable for surgical treatment. Palliative therapy including chemotherapy and best supportive care do not achieve satisfactory results (Wang et al., 2014a; Wang et al., 2014b). In patients treated by gastrectomy and chemotherapy, median survival time was reported to range from 2.9 to 11.8 months (Maehara et al., 1991; Okano et al., 2002). In comparison with other treatments, it appears that gastrectomy combined with hepatectomy has a beneficial effect on long-term prognosis. 
The first publication dedicated to the clinical outcome after liver resection for gastric cancer metastases was presented by Ochiai et al. (1994): they reported a median survival time of 18 months and an overall 5-year survival rate of 19\%. Qiu et al. (2013) reported that the median survival of 25 synchronous metastasis patients was 38.0 months in his study. Our long-term results are similar to those published by above authors; in our analysis of 35 patients who underwent simultaneous resection of gastric primary tumour and synchronous liver metastases, we found a median survival of 33 months and an overall 5 year survival rate of $14.3 \%$. The results of a metaanalysis including 19 studies have shown that the median survival for all 436 patients with liver resection for hepatic metastasized gastric cancer was 17 months, and the 5-year survival was $26.5 \%$ (Kerkar et al., 2010). However, Bines et al. (1993) have revealed that hepatic resection did not produce long-term survival in patients with synchronous metastases, because of the small number of patients in his study, it is difficult to introduce definitive conclusions.

Our univariate analysis revealed that the presence of lymphovascular invasion of the primary gastric tumor, bilateral liver metastasis and multiple liver metastasis are associated with poor survival for patients underwent simultaneous resection of gastric primary tumour and synchronous liver metastases. In contrast to our results, Ochiai et al. (1994) reported that the significant factor associated with survival was only the microscopic depth of invasion of primary tumor, and that hepatic resection should be attempted in patients with metastases if there is no serosal invasion of the primary gastric tumor. Some authors reported that the clinicopathological factors related to the primary gastric cancer, including depth of the primary tumour and lymph node metastasis, were significant prognostic factors for survival (Kumagai et al., 2001; Koga et al., 2007a; Sakamoto et al., 2007). Koga et al. (Koga et al., 2007b) reported 5-year survival rates of $55 \%$ for solitary metastasis and $0 \%$ for multiple metastases; a similar conclusion was confirmed by other studies as well (Okano et al., 2002; Sakamoto et al., 2003; Cheon et al., 2008; Garancini et al., 2012). Solitary metastases was significant determinants for a favorable prognosis after hepatic resection. A generous surgical margin may not be essential for curative hepatic resection of liver metastases, because recurrence is strongly associated with systemic spread rather than local invasion (Okano et al., 2002). However, we advise that the surgeon should obtain an adequate margin to the best of his ability if technically feasible. Sakamoto et al. (2007) demonstrated that small-sized liver metastases were associated with more favourable results than larger metastases following hepatic resection. However, our study showed no significant survival difference between those with liver metastases tumours $\leq 2.0 \mathrm{~cm}$ as compared with those $>2.0 \mathrm{~cm}$ in tumour diameter.

Indications for a simultaneous combined approach for synchronous hepatic metastases from gastric carcinoma have been rarely reported. This study identified the presence of lymphovascular invasion of the primary gastric tumor and multiple hepatic tumors as significant independent indicators of poor prognosis by multivariate analysis; nevertheless, it presents one of the largest retrospective case series studied so far. Our study indicated that patients with solitary liver metastasis and without lymphovascular invasion of the primary gastric tumor are the best candidates for liver resection. However, survival after resection of up to eight metastases is similar to that after resection of a solitary metastasis in colorectal liver metastases and the number of metastases is not important predictor of long-term survival (Okano et al., 2002).The different indications for resection of liver metastases between colorectal and gastric cancer are thought to reflect the aggressive biologic behavior of gastric cancer.

As the nature of our study is small and retrospective, a larger prospective series or a multi-centered approach may make more substantive conclusions. The surgical treatment of synchronous hepatic metastases from gastric carcinoma deserves to be concerned about and should be investigated in further analyses.

This study indicated that patients with solitary hepatic tumors and without lymphovascular invasion of the primary gastric tumor may be good candidates for a hepatectomy, and that liver resection may prolong the patients' survival.

\section{References}

Ambiru S, Miyazaki M, Ito H, et al (2001). Benefits and limits of hepatic resection for gastric metastases. Am J Surg, 181, 279-83.

Bines SD, England G, Deziel DJ, et al (1993). Synchronous, metachronous, and multiple hepatic resections of liver tumors originating from primary gastric tumors. Surgery, 114, 799-805; discussion 4-5.

Cheon SH, Rha SY, Jeung HC, et al (2008). Survival benefit of combined curative resection of the stomach (D2 resection) and liver in gastric cancer patients with liver metastases. Ann Oncol, 19, 1146-53.

Garancini M, Uggeri F, Degrate L, et al (2012). Surgical treatment of liver metastases of gastric cancer: is local treatment in a systemic disease worthwhile? HPB (Oxford), 14, 209-15.

Kerkar SP, Kemp CD, Avital I (2010). Liver resections in metastatic gastric cancer. $H P B(O x f o r d), 12,589-96$.

Koga R, Yamamoto J, Ohyama S, et al (2007a). Liver resection for metastatic gastric cancer: experience with 42 patients including eight long-term survivors. Jpn J Clin Oncol, 37, 836-42.

Koga R, Yamamoto J, Ohyama S, et al (2007b). Liver resection for metastatic gastric cancer: experience with 42 patients including eight long-term survivors. Jpn J Clin Oncol, 37, 836-42.

Kumagai K, Tanaka T, Yamagata K, et al (2001). Liver metastasis in gastric cancer with particular reference to lymphatic advancement. Gastric Cancer, 4, 150-5.

Maehara Y, Moriguchi S, Kakeji Y, et al 1991. Pertinent risk factors and gastric carcinoma with synchronous peritoneal dissemination or liver metastasis.

Mann CD, Neal CP, Pattenden CJ, et al (2008). Major resection of hepatic colorectal liver metastases in elderly patients an aggressive approach is justified. Eur J Surg Oncol, 34, 428-32.

Morise Z, Sugioka A, Hoshimoto S, et al (2008). The role of hepatectomy for patients with liver metastases of gastric cancer. Hepatogastroenterology, 55, 1238-41. 
Ochiai T, Sasako M, Mizuno S, et al (1994). Hepatic resection for metastatic tumours from gastric cancer: analysis of prognostic factors. Br J Surg, 81, 1175-8.

Okano K, Maeba T, Ishimura K, et al (2002). Hepatic resection for metastatic tumors from gastric cancer. Ann Surg, 235, 86-91.

Okano K, Yamamoto J, Moriya Y, et al (1999). Macroscopic intrabiliary growth of liver metastases from colorectal cancer. Surgery, 126, 829-34.

Qiu JL, Deng MG, Li W, et al (2013). Hepatic resection for synchronous hepatic metastasis from gastric cancer. Eur $J$ Surg Oncol, 39, 694-700.

Sakamoto Y, Ohyama S, Yamamoto J, et al (2003). Surgical resection of liver metastases of gastric cancer: an analysis of a 17-year experience with 22 patients. Surgery, 133, 507-11.

Sakamoto Y, Sano T, Shimada K, et al (2007). Favorable indications for hepatectomy in patients with liver metastasis from gastric cancer. J Surg Oncol, 95, 534-9.

Shirabe K, Shimada M, Matsumata T, et al (2003). Analysis of the prognostic factors for liver metastasis of gastric cancer after hepatic resection: a multi-institutional study of the indications for resection. Hepatogastroenterology, 50, 1560-3.

Thelen A, Jonas S, Benckert C, et al (2008). Liver resection for metastatic gastric cancer. Eur J Surg Oncol, 34, 1328-34.

Tiberio GA, Coniglio A, Marchet A, et al (2009). Metachronous hepatic metastases from gastric carcinoma: a multicentric survey. Eur J Surg Oncol, 35, 486-91.

Tsujimoto H, Ichikura T, Ono S, et al (2010). Outcomes for patients following hepatic resection of metastatic tumors from gastric cancer. Hepatol Int, 4, 406-13.

Wang JC, Tian JH, Ge L, et al (2014a). Which is the best Chinese herb injection based on the FOLFOX regimen for gastric cancer? A network meta- analysis of randomized controlled trials. Asian Pac J Cancer Prev, 15, 4795-800.

Wang Z, Ma L, Zhang XM, et al (2014b). Long-term outcomes after D2 gastrectomy for early gastric cancer: survival analysis of a single-center experience in China. Asian Pac J Cancer Prev, 15, 7219-22.

Yasui K, Hirai T, Kato T, et al (1997). A new macroscopic classification predicts prognosis for patient with liver metastases from colorectal cancer. Ann Surg, 226, 582-6.

Zhang XM, Wang Z, Liang JW, et al (2014). Analysis of laparoscopy-assisted gastric cancer operations performed by inexperienced junior surgeons. Asian Pac J Cancer Prev, $15,5077-81$. 